\title{
ON THE REALIZATION THEORY OF POLYNOMIAL MATRICES AND THE ALGEBRAIC STRUCTURE OF PURE GENERALIZED STATE SPACE SYSTEMS
}

\author{
ANTONIS - IOANNIS G. VARDULAKIS *, NiCHOLAS P. KARAMPETAKIS *, \\ EFstATHIOS N. ANTONIOU ${ }^{* *}$, EVANGELIA TICTOPOULOU ${ }^{* * *}$ \\ * Department of Mathematics \\ Aristotle University of Thessaloniki, Thessaloniki 54 006, Greece \\ e-mail: \{avardula, karampet\} @math.auth.gr \\ ** Department of Sciences \\ Technical Educational Institute of Thessaloniki, Sindos 574 00, Greece \\ e-mail: eantonio@gen.teithe.gr \\ *** General Department of Applied Science \\ Technical University of Chalkis, Psahna 34 400, Eubea, Greece \\ e-mail: etikt@teihal.gr
}

\begin{abstract}
We review the realization theory of polynomial (transfer function) matrices via "pure" generalized state space system models. The concept of an irreducible-at-infinity generalized state space realization of a polynomial matrix is defined and the mechanism of the "cancellations" of "decoupling zeros at infinity" is closely examined. The difference between the concepts of irreducibility and minimality of generalized state space realizations of polynomial (transfer function) matrices is pointed out and the associated concepts of dynamic and non-dynamic variables appearing in generalized state space realizations are also examined.
\end{abstract}

Keywords: polynomial matrices, realization theory, minimality, irreducibility, generalized state space, infinite decoupling zeros.

\section{Introduction}

Starting with Rosenbrock's seminal paper (Rosenbrock, 1974), the algebraic structure of generalized state space (GSS) or singular systems and the associated problems dealing with the realization theory of non-proper real rational matrices has been the subject of numerous and important investigations during the past 35 years (Bosgra and Van Der Weiden, 1981; Verghese, 1978; Verghese et al., 1981; Cobb, 1984; Lewis, 1986; Lewis et al., 1989; Conte and Perdon, 1982; Misra and Patel, 1989; Christodoulou and Mertzios, 1986; Vafiadis and Karcanias, 1995). In this paper, motivated by the classical realization theory of proper rational transfer function matrices of linear time invariant multivariable systems by ordinary state space system models, we examine some ideas related to the realization theory of polynomial transfer function matrices that correspond to linear, time invariant, "pure” general- ized state space systems along with the associated concepts of "decoupling zeros at infinity" and minimality and irreducibility of such systems.

Although the concepts of reducibility or irreducibility "at infinity" and "decoupling zeros at infinity" of generalized state space realizations of polynomial matrices are implicit in many papers, they have not been clearly defined. In most cases (see, e.g., (Bosgra and Van Der Weiden, 1981; Varga, 1989; Misra and Patel, 1989; Vafiadis and Karcanias, 1995)), the authors focus on the computational aspects of the reduction techniques introduced, without going into details regarding the action of these methods on the underlying algebraic structure of the matrices involved. Similarly, the mechanism of the "cancellations" of "decoupling zeros at infinity" during the formation of the polynomial transfer function matrix from a reducible-at-infinity generalized state space realization of such a polynomial matrix has not been clearly explained 
in all of the above studies and is closely examined, we believe for the first time, here.

Our approach focuses on the investigation of the algebraic structure of the matrices describing "pure"generalized state space systems. The difference between the concepts of irreducibility at infinity and minimality of "pure" generalized state space realizations of polynomial (transfer function) matrices is pointed out and the relations of these concepts with the associated concepts of dynamic and non-dynamic variables appearing in such generalized state space realizations are also reviewed.

The paper is organized as follows: In Section 2, we give a brief review of known results from the theory of polynomial matrices which will be useful in the sequel. In Section 3, we investigate the concept of irreducibility at infinity, while in Section 4 , we present the difference between the concepts of irreducibility "at infinity" and minimality which depends on the presence of non-dynamic variables. Finally, in Section 5, we summarize our results and give some conclusions.

\section{Mathematical background}

In what follows, the time variable $t$ is considered to be continuous, i.e., taking values in $\mathbb{R}$. Correspondingly, the variable $s$ can be considered as denoting the Laplace variable in the Laplace transform $\mathcal{L}\{x(t)\}=X(s):=$ $\int_{0^{-}}^{\infty} x(t) e^{-s t} \mathrm{~d} t$ of a continuous time function $x(t): \mathbb{R} \rightarrow$ $\mathbb{R}$. By $\mathbb{R}(s)^{p \times m}, \mathbb{R}_{p r}(s)^{p \times m}$ and $\mathbb{R}[s]^{p \times m}$ we denote respectively the sets of $p \times m$ rational, proper rational and polynomial matrices with real coefficients and indeterminate $s \in \mathbb{C}$. A polynomial matrix

$$
A(s)=A_{q} s^{q}+A_{q-1} s^{q-1}+\cdots+A_{0},
$$

where $A_{k} \in \mathbb{R}^{p \times m}, k=0,1, \ldots, q \geq 1, A_{q} \neq 0$, is called regular iff $p=m$ and $\operatorname{det} A(s) \neq 0$ for almost every $s \in \mathbb{C}$. In any other case, i.e., if $p \neq m$ or $p=m$ and $\operatorname{det} A(s)=0$, it is called singular. If $q=1$, then $A(s)=A_{1} s+A_{0} \in \mathbb{R}[s]^{p \times m}$ is called a matrix pencil (Gantmacher, 1959). The (finite) zeros of $A(s)$ are defined as the roots of the equation $\operatorname{det} A(s)=0$. Equivalently, $\lambda_{i} \in \mathbb{C}$ is a (finite) zero of $A(s)$ iff $\operatorname{rank}_{\mathbb{C}} A\left(\lambda_{i}\right)<r$. $\delta_{M}[\cdot]$ denotes the McMillan degree of a rational matrix, i.e., the total number of its poles in $\mathbb{C} \cup\{\infty\}$. Every rational matrix $A(s) \in \mathbb{R}(s)^{p \times m}$ with $\operatorname{rank}_{\mathbb{R}(s)} A(s)=r \leq$ $\min (p, m)$ is biproperly equivalent (Vardulakis, 1991) to its Smith-McMillan form at $s=\infty$,

$$
\begin{aligned}
S_{A(s)}^{\infty}= & \operatorname{diag}(\overbrace{k}^{\overbrace{s^{q_{1}}, \ldots, s^{q_{k}}}^{v}, I_{v-k}}, \\
& \overbrace{\frac{1}{s^{\widehat{q}_{v+1}}}, \ldots, \frac{1}{s^{\widehat{q}_{r}}}}^{r-v}, 0_{p-r, m-r}),
\end{aligned}
$$

where $r \geq v>k \geq 0$ and $q_{1}>q_{2}>\ldots>q_{k}>$ $0=q_{k+1}=\cdots=q_{v}, \widehat{q}_{r} \geq \widehat{q}_{r-1} \geq \cdots \geq \widehat{q}_{v+1}>0$ are respectively the orders of the poles and the zeros of $A(s)$ at $s=\infty$. Finally, if $A(s)$ is as in (1) with the Smith-McMillan form at $s=\infty$ as in (2), then it turns out (Vardulakis et al., 1982) that

$$
q=q_{1} .
$$

We also give a review of some known facts and basic results regarding the "realization theory" of polynomial matrices. What follows can be seen as an extension of the results regarding the realization theory of proper rational matrices to the case of polynomial matrices .

Definition 1. Let $A(s) \in \mathbb{R}[s]^{p \times m}, \operatorname{rank}_{\mathbb{R}(s)} A(s)=$ $r \leq \min (p, m)$. A quadruple of matrices $C_{\infty} \in$ $\mathbb{R}^{p \times \mu}, A_{\infty} \in \mathbb{R}^{\mu \times \mu}, B_{\infty} \in \mathbb{R}^{\mu \times m}, D_{\infty} \in \mathbb{R}^{p \times m}, \mu \in$ $\mathbb{Z}^{+}$is called a generalized state space (GSS) realization of $A(s)$ iff the GSS system, denoted by $\Sigma_{g}$, is defined by

$$
\begin{aligned}
A_{\infty} \dot{x}_{\infty}(t) & =x_{\infty}(t)-B_{\infty} u(t), \\
y(t) & =C_{\infty} x_{\infty}(t)+D_{\infty} u(t),
\end{aligned}
$$

and the transfer function matrix between $Y(s)=\mathcal{L}\{y(t)\}$ and $U(s)=\mathcal{L}\{u(t)\}$ is the polynomial matrix $A(s)$, i.e., if

$$
A(s)=C_{\infty}\left(I_{\mu}-s A_{\infty}\right)^{-1} B_{\infty}+D_{\infty} .
$$

The vector $x_{\infty}(t): \mathbb{R} \rightarrow \mathbb{R}^{\mu}$ in (4) is called the (fast) generalized state vector of $\Sigma_{g}$ and the positive integer $\mu$ is called the dimension of $\Sigma_{g}$.

Remark 1. A GSS realization of $A(s) \in \mathbb{R}[s]^{p \times m}$ can always be obtained from a state space realization of the strictly proper rational matrix (Verghese, 1978),

$$
\bar{A}(s):=\left(\frac{1}{s}\right) A\left(\frac{1}{s}\right) \in \mathbb{R}_{p r}^{p \times m}(s)
$$

because if $C_{\infty} \in \mathbb{R}^{p \times \mu}, A_{\infty} \in \mathbb{R}^{\mu \times \mu}, B_{\infty} \in \mathbb{R}^{\mu \times m}$ is a state space realization of $\bar{A}(s)$, i.e., if

$$
\left(\frac{1}{s}\right) A\left(\frac{1}{s}\right)=C_{\infty}\left(s I_{\mu}-A_{\infty}\right)^{-1} B_{\infty},
$$

then (7), by the substitution $1 / s \mapsto s$, gives (6) with $D_{\infty}=0_{p, m}$.

Let $A(s)=A_{0}+A_{1} s+\cdots+A_{q_{1}} s^{q_{1}} \in \mathbb{R}[s]^{p \times m}$, $A_{i} \in \mathbb{R}^{p \times m}, i=0,1,2, \ldots, q_{1} \geq 1, A_{q_{1}} \neq 0$ and let $C_{\infty} \in \mathbb{R}^{p \times \mu}, A_{\infty} \in \mathbb{R}^{\mu \times \mu}, B_{\infty} \in \mathbb{R}^{\mu \times m}, D_{\infty} \in \mathbb{R}^{p \times m}$, $\mu \in \mathbb{Z}^{+}$be a GSS realization of $A(s)$. Let also $J_{\infty}=$ $Q A_{\infty} Q^{-1}, Q \in \mathbb{R}^{\mu \times \mu},|Q| \neq 0$, be the Jordan normal form of $A_{\infty}$, and $\bar{C}_{\infty}:=C_{\infty} Q^{-1}, \bar{B}_{\infty}:=Q B_{\infty}$. From (6) it follows that $\left(I_{\mu}-s A_{\infty}\right)^{-1} \in \mathbb{R}[s]^{\mu \times \mu}$, so that $I_{\mu}-s A_{\infty}$ or, equivalently, $I_{\mu}-s J_{\infty}$ are $\mathbb{R}[s]$-unimodular matrices and $J_{\infty}$ has, in general, the form

$$
J_{\infty}=\text { block diag }\left[J_{\infty 1}, J_{\infty 2}, \ldots, J_{\infty \eta}, 0_{\tau, \tau}\right] \in \mathbb{R}^{\mu \times \mu},
$$


where

$$
J_{\infty i}=\left[\begin{array}{cccc}
0 & 1 & \ldots & 0 \\
0 & \ddots & \ddots & \vdots \\
\vdots & \ddots & \ddots & 1 \\
0 & \ldots & 0 & 0
\end{array}\right] \in \mathbb{R}^{\left(\kappa_{i}+1\right) \times\left(\kappa_{i}+1\right)}
$$

and $\kappa_{1} \geq \kappa_{2} \geq \cdots \geq \kappa_{\eta}, \kappa_{i} \in \mathbb{Z}^{+}, i=1,2, \ldots, \eta$. From (8) and (9) it follows that $J_{\infty}$ (equivalently $A_{\infty}$ ) is a nilpotent matrix with the index of nilpotency equal to the size $\kappa_{1}+1$ of its largest Jordan block $J_{\infty 1}$, i.e., $J_{\infty}^{\kappa_{1}} \neq 0_{\mu, \mu}, J_{\infty}^{\kappa_{1}+i}=0_{\mu, \mu}, i=1,2, \ldots$, and it can be easily verified that

$$
\left(I_{\mu}-s J_{\infty}\right)^{-1}=I_{\mu}+s J_{\infty}+\cdots+s^{\kappa_{1}} J_{\infty}^{\kappa_{1}} \in \mathbb{R}[s]^{\mu \times \mu} .
$$

From the fact that

$$
A(s)=\bar{C}_{\infty}\left(I_{\mu}-s J_{\infty}\right)^{-1} \bar{B}_{\infty}+D_{\infty}
$$

and (10) it follows that $\kappa_{1} \geq q_{1}$ and

$$
\begin{aligned}
\bar{C}_{\infty} J_{\infty}^{i} \bar{B}_{\infty}=A_{i}, & i=0,1,2, \ldots, q_{1}, \\
\bar{C}_{\infty} J_{\infty}^{i} \bar{B}_{\infty}=0, & i=q_{1}+1, q_{1}+2, \ldots
\end{aligned}
$$

We give now a number of definitions and results regarding the structure of the GSS realization of a polynomial matrix $A(s)$.

Definition 2. The GSS system poles at $s=\infty$ of the GSS realization $\Sigma_{g}=\left[C_{\infty}, A_{\infty}, B_{\infty}, D_{\infty}\right]$ of $A(s)$ are the zeros at $s=\infty$ of $I_{\mu}-s A_{\infty}$. The generalized order $f_{g}$ of $\Sigma_{g}$ is the total number of the system poles at $s=\infty$ of $\Sigma_{g}$ or, equivalently, the total number of zeros at $s=\infty$ of $I_{\mu}-s A_{\infty}$. (The multiplicities and orders of the zeros at $s=\infty$ of $I_{\mu}-s A_{\infty}$ are accounted for.)

From (8) and (9) it can be easily seen that the orders of the zeros at $s=\infty$ of $I_{\mu}-s J_{\infty}$ are the integers $\kappa_{i} \geq 1, i=$ $1,2, \ldots, \eta$, i.e., that the Smith-McMillan form $S_{I_{\mu}-s J_{\infty}}^{\infty}$ at $s=\infty$ of the matrix pencil $I_{\mu}-s A_{\infty}$ (Vardulakis and Karcanias, 1983; Vardulakis, 1991) is given by

$$
S_{I_{\mu}-s J_{\infty}}^{\infty}=\operatorname{block} \operatorname{diag}\left[s I_{f_{g}}, I_{\tau}, \frac{1}{s^{\kappa_{\eta}}}, \ldots, \frac{1}{s^{\kappa_{1}}}\right] .
$$

Now, since $I_{\mu}-s A_{\infty}$ is a unimodular polynomial matrix (in fact, a regular matrix pencil), it has no finite zeros, i.e., the number of the finite zeros of $I_{\mu}-s A_{\infty}$ is $n:=\operatorname{deg}\left|I_{\mu}-s A_{\infty}\right|=0$ and has no finite poles. Now, from the known fact that in every square and nonsingular polynomial matrix $A(s)$ the total number of zeros of $A(s)$ in $\mathbb{C} \cup\{\infty\}$ is equal to the total number of poles of $A(s)$ in $\mathbb{C} \cup\{\infty\}$ (Vardulakis, 1991), it simply follows that the generalized order $f_{g}$ of $\Sigma_{g}$ is given also by the total number of poles at $s=\infty$ of $I_{\mu}-s A_{\infty}$ which, by definition, is its McMillan degree $\delta_{M}\left[I_{\mu}-s A_{\infty}\right]$. Summarizing, we have that

$$
\begin{aligned}
f_{g} & :=\text { total number of zeros at } s=\infty \text { of }\left[I_{\mu}-s A_{\infty}\right] \\
& \stackrel{13}{=} \sum_{i=1}^{\eta} \kappa_{i}=\operatorname{rank}_{\mathbb{R}} J_{\infty}=\operatorname{rank}_{\mathbb{R}} A_{\infty} \\
& =\text { total \# of poles at } s=\infty \text { of }\left[I_{\mu}-s A_{\infty}\right] \\
& =: \delta_{M}\left[I_{\mu}-s A_{\infty}\right]
\end{aligned}
$$

where the symbol '\#' means the "total number" with multiplicities accounted for.

\section{Irreducibility at infinity}

We examine now the concept of irreducibility at $s=\infty$ of a GSS realization of a polynomial matrix. This concept is analogous to that of irreducibility in $\mathbb{C}$ of a state space realization of a proper rational matrix. To this end, we introduce a number of auxiliary results.

Let $A(s) \in \mathbb{R}[s]^{p \times m}$, and let $\bar{C}_{\infty} \in \mathbb{R}^{p \times \mu}, J_{\infty} \in$ $\mathbb{R}^{\mu \times \mu}, \bar{B}_{\infty} \in \mathbb{R}^{\mu \times m}, D_{\infty} \in \mathbb{R}^{p \times m}, \mu \in \mathbb{Z}^{+}$be a GSS realization of $A(s)$ with $J_{\infty}$ in Jordan normal form as in (8) and (9), so that $\mu:=\sum_{i=1}^{\eta}\left(\kappa_{i}+1\right)+\tau$. Let

$$
\begin{aligned}
& \bar{C}_{\infty}=\left[\begin{array}{llll}
C_{\infty 1} & C_{\infty 2} & \ldots & C_{\infty \eta} \\
C_{\infty \eta+1}
\end{array}\right] \in \mathbb{R}^{p \times \mu}, \\
& C_{\infty i}=\left[\begin{array}{lllll}
c_{i 1} & c_{i 2} & \ldots & c_{i \kappa_{i}} & c_{i \kappa_{i}+1}
\end{array}\right] \in \mathbb{R}^{p \times\left(\kappa_{i}+1\right)},
\end{aligned}
$$

where $c_{i j} \in \mathbb{R}^{p \times 1}, i=1,2, \ldots, \eta, j=1,2, \ldots, \kappa_{i}+1$. Let also

$$
\begin{aligned}
\bar{B}_{\infty}= & {\left[\begin{array}{l}
B_{\infty 1} \\
\vdots \\
B_{\infty \eta} \\
B_{\infty \eta+1}
\end{array}\right] \in \mathbb{R}^{\mu \times m}, } \\
B_{\infty i}= & {\left[\begin{array}{l}
b_{i 1}^{\top} \\
\vdots \\
b_{i \kappa_{i}}^{\top} \\
b_{i \kappa_{i}+1}^{\top}
\end{array}\right] \in \mathbb{R}^{\left(\kappa_{i}+1\right) \times m}, }
\end{aligned}
$$

with $b_{i j}^{\top} \in \mathbb{R}^{1 \times m}, i=1,2, \ldots, \eta$ and $j=1,2, \ldots, \kappa_{i}+1$. Consider the singular matrix pencils

$$
\begin{aligned}
& {\left[\begin{array}{c}
I_{\kappa_{i}+1}-s J_{\infty i} \\
C_{\infty i}
\end{array}\right]=\left[\begin{array}{l}
I_{\kappa_{i}+1} \\
C_{\infty i}
\end{array}\right]-s\left[\begin{array}{l}
J_{\infty i} \\
0
\end{array}\right],} \\
& {\left[\begin{array}{cc}
I_{\kappa_{i}+1}-s J_{\infty i} & B_{\infty i}
\end{array}\right]} \\
& =\left[\begin{array}{ll}
I_{\kappa_{i}+1} & B_{\infty i}
\end{array}\right]-s\left[\begin{array}{ll}
J_{\infty i} & 0
\end{array}\right],
\end{aligned}
$$

where $i=1,2, \ldots, \eta$. 


\section{Proposition 1.}

(i) The singular matrix pencil

$$
\left[\begin{array}{c}
I_{\kappa_{i}+1}-s J_{\infty i} \\
C_{\infty i}
\end{array}\right]
$$

has no zeros at $s=\infty$ iff $c_{i 1} \neq 0$ or, equivalently,

$$
\operatorname{rank}_{\mathbb{R}}\left[\begin{array}{c}
J_{\infty i} \\
C_{\infty i}
\end{array}\right]=\kappa_{i}+1
$$

(ii) The singular matrix pencil

$$
\left[\begin{array}{ll}
I_{\kappa_{i}+1}-s J_{\infty i} & B_{\infty i}
\end{array}\right]
$$

has no zeros at $s=\infty$ iff $b_{i \kappa_{i}+1}^{\top} \neq 0$ or, equivalently,

$$
\operatorname{rank}_{\mathbb{R}}\left[J_{\infty i}, B_{\infty i}\right]=\kappa_{i}+1 .
$$

Proof. We prove the first assertion. The second assertion can be proved in a similar way. The singular matrix pencil in (19) can be written as

$$
\begin{aligned}
& {\left[\begin{array}{c}
I_{\kappa_{i}+1}-s J_{\infty i} \\
C_{\infty i}
\end{array}\right]=\left[\begin{array}{ccccc}
1 & -1 & 0 & \ldots & 0 \\
0 & \frac{1}{s} & -1 & \ldots & 0 \\
\vdots & \ddots & \ddots & \ddots & \vdots \\
0 & 0 & \ddots & \ddots & -1 \\
0 & 0 & 0 & \ldots & \frac{1}{s} \\
---\frac{\bar{c}}{c_{i 2}} & \frac{\bar{c}_{i 3}}{c_{i 1}} & \ldots & \ldots & \frac{c_{i \kappa_{i}+1}}{s}
\end{array}\right]} \\
& \times\left[\begin{array}{cccc}
1 & 0 & \ldots & 0 \\
0 & \frac{1}{s} & \ldots & 0 \\
\vdots & \vdots & \ddots & \vdots \\
0 & 0 & \ldots & \frac{1}{s}
\end{array}\right]^{-1} \\
& =: N_{i}(s) D_{i}(s)^{-1}
\end{aligned}
$$

and, since

$$
\operatorname{rank}_{\mathbb{R}}\left[\begin{array}{c}
N_{i}(\infty) \\
D_{i}(\infty)
\end{array}\right]=\kappa_{i}+1
$$

(23) is a coprime-at-s $=\infty$ proper rational matrix fractional representation of (19), see (Vardulakis, 1991). In (Vardulakis, 1991) it is proved that the zeros at $s=\infty$ of 19$)$ considered as an element of $\mathbb{R}(s)^{\left(\kappa_{i}+1+p\right) \times\left(\kappa_{i}+1\right)}$ are given by the zeros at $s=\infty$ of the numerator $N_{i}(s) \in \mathbb{R}_{p r}(s)^{\left(\kappa_{i}+1+p\right) \times\left(\kappa_{i}+1\right)}$ in 23) which has no zeros at $s=\infty$ iff

$$
\operatorname{rank}_{\mathbb{R}} N_{i}(\infty)=\kappa_{i}+1,
$$

which is clearly equivalent to 20).
Using arguments similar to those in the proof of Proposition 1, we can easily show the following result.

\section{Corollary 1. Let}

$$
C_{\infty i}=\left[c_{i 1}, \ldots, c_{i \kappa_{i}}, c_{i \kappa_{i}+1}\right] \in \mathbb{R}^{p \times\left(\kappa_{i}+1\right)}
$$

such that

$c_{i 1}=\cdots=c_{i \sigma_{i}-1}=0, \quad c_{i \sigma_{i}} \neq 0, \quad 1 \leq \sigma_{i} \leq \kappa_{i}+1$.

Then

$$
\left[\begin{array}{c}
I_{\kappa_{i}+1}-s J_{\infty i} \\
C_{\infty i}
\end{array}\right] \in \mathbb{R}^{\left(\kappa_{i}+1+p\right) \times\left(\kappa_{i}+1\right)}
$$

has one zero at $s=\infty$ of order $\sigma_{i}-1$. By analogy, a similar result holds for the pencil $\left[\begin{array}{ll}I_{\kappa_{i}+1}-s J_{\infty i} & B_{\infty i}\end{array}\right]$.

Due to the Jordan block structure of $J_{\infty} \in \mathbb{R}^{\mu \times \mu}$ as in (8) and the block structures of $\bar{C}_{\infty}$ and $\bar{B}_{\infty}$ as in (15) (17), the above corollary implies also the following result.

Corollary 2. Let $A(s) \in \mathbb{R}[s]^{p \times m}$ and $\bar{C}_{\infty} \in$ $\mathbb{R}^{p \times \mu}, J_{\infty} \in \mathbb{R}^{\mu \times \mu}, \bar{B}_{\infty} \in \mathbb{R}^{\mu \times m}, D_{\infty} \in \mathbb{R}^{p \times m}, \mu \in$ $Z^{+}$be a GSS realization of $A(s)$ with $J_{\infty}$ in the Jordan normal form as in (8) and (9). Then

(i) The matrix

$$
\left[\begin{array}{c}
I_{\mu}-s J_{\infty i} \\
\bar{C}_{\infty}
\end{array}\right]
$$

has no zeros at $s=\infty$ iff

$$
\operatorname{rank}_{\mathbb{R}}\left[\begin{array}{c}
J_{\infty} \\
\bar{C}_{\infty}
\end{array}\right]=\mu
$$

(ii) $\left[\begin{array}{cc}I_{\mu}-s J_{\infty i} & \bar{B}_{\infty}\end{array}\right]$ has no zeros at $s=\infty$ iff $\operatorname{rank}_{\mathbb{R}}\left[\begin{array}{cc}J_{\infty} & \bar{B}_{\infty}\end{array}\right]=\mu$.

A direct consequence of the above results is also the following result.

Corollary 3. $C_{\infty i} J_{\infty i}=0 \Leftrightarrow c_{i 1}=c_{i 2}=\cdots=c_{i \kappa_{i}}=$ $0 \stackrel{\text { Cor. } 1}{\Rightarrow}$ the matrix

$$
\left[\begin{array}{c}
I_{\kappa_{i}+1}-s J_{\infty i} \\
C_{\infty i}
\end{array}\right]
$$

has one zero at $s=\infty$ of order $\kappa_{i}$.

In order now to proceed with the concept of irreducibility at infinity of a GSS realization of a polynomial matrix, consider the following motivating example.

Example 1. Let $\bar{C}_{\infty} \in \mathbb{R}^{p \times \mu}, J_{\infty} \in \mathbb{R}^{\mu \times \mu}, \bar{B}_{\infty} \in$ $\mathbb{R}^{\mu \times m}, D_{\infty} \in \mathbb{R}^{p \times m}$ be a GSS realization of a polynomial matrix $A(s) \in \mathbb{R}[s]^{p \times m}$. For simplicity of notation, set $\eta=3$,

$$
\bar{C}_{\infty}=\left[\begin{array}{llll}
C_{\infty 1} & C_{\infty 2} & C_{\infty 3} & C_{\infty 4}
\end{array}\right],
$$


where $C_{\infty i} \in \mathbb{R}^{p \times\left(\kappa_{i}+1\right)}, i=1,2,3, C_{\infty 4} \in \mathbb{R}^{p \times \tau}$,

$$
J_{\infty}=\text { block diag }\left[J_{\infty 1}, J_{\infty 2}, J_{\infty 3}, 0_{\tau, \tau}\right] \in \mathbb{R}^{\mu \times \mu},
$$

$$
\bar{B}_{\infty}=\left[\begin{array}{c}
B_{\infty 1} \\
B_{\infty 2} \\
B_{\infty 3} \\
B_{\infty 4}
\end{array}\right],
$$

$B_{\infty i} \in \mathbb{R}^{\left(\kappa_{i}+1\right) \times m}, i=1,2,3, B_{\infty 4} \in \mathbb{R}^{\tau \times m}$ so that the generalized order of $\Sigma_{g}=\left[\bar{C}_{\infty}, J_{\infty}, \bar{B}_{\infty}, D_{\infty}\right]$ is $f_{g}=\operatorname{rank}_{\mathbb{R}} J_{\infty}=\sum_{i=1}^{3} \kappa_{i}$. Notice that $A(s)$ can be written as

$$
\begin{aligned}
A(s)= & \bar{C}_{\infty}\left(I_{\mu}-s J_{\infty}\right)^{-1} \bar{B}_{\infty}+D_{\infty} \\
= & C_{\infty 1}\left[I_{\kappa_{1}+1}-s J_{\infty 1}\right]^{-1} B_{\infty 1} \\
& +C_{\infty 2}\left[I_{\kappa_{2}+1}-s J_{\infty 2}\right]^{-1} B_{\infty 2} \\
& +C_{\infty 3}\left[I_{\kappa_{3}+1}-s J_{\infty 3}\right]^{-1} B_{\infty 3} \\
& +C_{\infty 4} B_{\infty 4}+D_{\infty} .
\end{aligned}
$$

Assume now for notational simplicity that for some $i \in\{1,2,3\}$ we have that

$$
C_{\infty i}=C_{\infty i}\left[I_{\kappa_{i}+1}-s J_{\infty i}\right] .
$$

Then by Corollary 3 this assumption implies that the polynomial matrix

$$
\left[\begin{array}{c}
I_{\kappa_{i}+1}-s J_{\infty i} \\
C_{\infty i}
\end{array}\right]
$$

has a zero of order $\kappa_{i}$ at $s=\infty$ which is the zero at $s=\infty$ of $I_{\kappa_{i}+1}-s J_{\infty i}$. For example, assume that (25) holds true for $i=2$. Then we will have

$$
\left[\begin{array}{c}
I_{\kappa_{2}+1}-s J_{\infty 2} \\
C_{\infty 2}
\end{array}\right]=\left[\begin{array}{c}
I_{\kappa_{2}+1} \\
C_{\infty 2}
\end{array}\right]\left[I_{\kappa_{2}+1}-s J_{\infty 2}\right]
$$

and from 25)

$$
C_{\infty 2}\left[I_{\kappa_{2}+1}-s J_{\infty 2}\right]^{-1} B_{\infty 2}=C_{\infty 2} B_{\infty 2},
$$

so that

$$
\begin{aligned}
A(s) & =\left[\begin{array}{ll}
C_{\infty 1} & C_{\infty 3}
\end{array}\right] \\
& \times\left[\begin{array}{ll}
I_{\kappa_{1}+1}-s J_{\infty 1} & 0 \\
0 & I_{\kappa_{3}+1}-s J_{\infty 3}
\end{array}\right]^{-1}\left[\begin{array}{l}
B_{\infty 1} \\
B_{\infty 3}
\end{array}\right] \\
& +C_{\infty 2} B_{\infty 2}+C_{\infty 4} B_{\infty 4}+D_{\infty} \\
& =\widehat{C}_{\infty}\left(I_{\mu}-s \widehat{J}_{\infty}\right)^{-1} \widehat{B}_{\infty}+\widehat{D}_{\infty},
\end{aligned}
$$

where

$$
\begin{aligned}
\widehat{C}_{\infty} & :=\left[\begin{array}{cc}
C_{\infty 1} & C_{\infty 3}
\end{array}\right] \in \mathbb{R}^{p \times \widehat{\mu}}, \\
\widehat{J}_{\infty} & :=\text { block diag }\left[J_{\infty 1}, J_{\infty 3}\right] \in \mathbb{R}^{\widehat{\mu} \times \widehat{\mu}}, \\
\widehat{B}_{\infty} & :=\left[\begin{array}{c}
B_{\infty 1} \\
B_{\infty 3}
\end{array}\right] \in \mathbb{R}^{\widehat{\mu} \times m}, \\
\widehat{D}_{\infty} & :=C_{\infty 2} B_{\infty 2}+C_{\infty 4} B_{\infty 4}+D_{\infty}, \\
\widehat{\mu} & =\left(\kappa_{1}+1\right)+\left(\kappa_{3}+1\right) .
\end{aligned}
$$

From the above example it follows that if, for some $i=1,2, \ldots, \eta$, the matrix pencil

$$
\left[\begin{array}{c}
I_{\kappa_{i}+1}-s J_{\infty i} \\
C_{\infty i}
\end{array}\right]
$$

has zeros of order $\kappa_{i}$ at $s=\infty$, then when $\bar{C}_{\infty}\left(I_{\mu}-s J_{\infty}\right)^{-1}$ is formed the system poles at $s=\infty$ of the GSS realization $\Sigma_{g}=\left[\bar{C}_{\infty}, J_{\infty}, \bar{B}_{\infty}, D_{\infty}\right]$ that correspond to the zero at $s=\infty$ of

$$
\left[\begin{array}{c}
I_{\kappa_{i}+1}-s J_{\infty i} \\
C_{\infty i}
\end{array}\right]
$$

of order $\kappa_{i}$ "cancel out" and they do not appear as poles at $s=\infty$ of the polynomial matrix $A(s)=$ $\bar{C}_{\infty}\left(I_{\mu}-s J_{\infty}\right)^{-1} \bar{B}_{\infty}$ (see $(25)$ ). Thus a GSS realization

$$
\widehat{\Sigma}_{g}=\left[\widehat{C}_{\infty}, \widehat{J}_{\infty}, \widehat{B}_{\infty}, \widehat{D}_{\infty}\right]
$$

of $A(s)$ is obtained which has generalized order $\widehat{f}_{g}:=$ $\kappa_{1}+\kappa_{3}<f_{g}$. Similar remarks apply if for some $i \in$ $\{1,2,3\}$ the matrix pencil $\left[I_{\kappa_{i}+1}-s J_{\infty i}, B_{\infty i}\right]$ has zeros at $s=\infty$.

The analysis in the above example gives rise to the concept of decoupling zeros at $s=\infty$ of a GSS realization $\Sigma_{g}=\left[C_{\infty}, A_{\infty}, B_{\infty}, D_{\infty}\right]$ of a polynomial matrix $A(s)$.

Definition 3. The input decoupling zeros (i.d.z.) (output decoupling zeros (o.d.z.)) at $s=\infty$ of a GSS realization $\Sigma_{g}=\left[C_{\infty}, A_{\infty}, B_{\infty}, D_{\infty}\right]$ of a polynomial matrix $A(s)$ are the zeros at $s=\infty$ of the singular matrix pencil $\left[I_{\mu}-s A_{\infty}, B_{\infty}\right]\left(\left[\begin{array}{c}{[c] c I_{\mu}-s A_{\infty}} \\ C_{\infty}\end{array}\right]\right)$. The input-output decoupling zeros (i.o.d.z.) at $s=\infty$ of $\Sigma_{g}$ are the common zeros at $s=\infty$ of the singular matrix pencils

$$
\left[I_{\mu}-s A_{\infty}, B_{\infty}\right], \quad\left[\begin{array}{c}
I_{\mu}-s A_{\infty} \\
C_{\infty}
\end{array}\right] .
$$

The decoupling zeros (d.z.) at $s=\infty$ of $\Sigma_{g}$ are the elements of the set

$$
\begin{aligned}
\left\{i . d . z . \text { ats }=\infty \text { of } \Sigma_{g}\right\} & \\
& +\left\{\text { o.d.z.ats }=\infty \text { of } \Sigma_{g}\right\} \\
& -\left\{\text { i.o.d.zerosats }=\infty \text { of } \Sigma_{g}\right\} .
\end{aligned}
$$

Candidates for (i.d.z.) and (o.d.z) at $s=\infty$ of a GSS realization of $\Sigma_{g}=\left[C_{\infty}, A_{\infty}, B_{\infty}, D_{\infty}\right]$ of a polynomial matrix $A(s)$ are the zeros at $s=\infty$ of $I_{\mu}-s A_{\infty}$, i.e., the system poles of $\Sigma_{g}$ at $s=\infty$.

Definition 4. A GSS realization

$$
\widehat{\Sigma}_{g}=\left[C_{\infty}, A_{\infty}, B_{\infty}, D_{\infty}\right]
$$

of a polynomial matrix $A(s)$ which has no input and no output decoupling zeros at $s=\infty$ is called an irreducible at $s=\infty$ GSS realization of $A(s)$. 
From Corollary 2 we obtain the following result.

Proposition 2. A GSS realization

$$
\begin{aligned}
\Sigma_{g}= & {\left[\bar{C}_{\infty} \in \mathbb{R}^{p \times \mu}, J_{\infty} \in \mathbb{R}^{\mu \times \mu}, \bar{B}_{\infty} \in \mathbb{R}^{\mu \times m},\right.} \\
& \left.D_{\infty} \in \mathbb{R}^{p \times m}\right]
\end{aligned}
$$

of a polynomial matrix $A(s)$ has no input and no output decoupling zeros at $s=\infty$, i.e., it is irreducible at $s=\infty$ or, equivalently, $\left[I_{\mu}-s J_{\infty}, \bar{B}_{\infty}\right]$ and

$$
\left[\frac{I_{\mu}-s J_{\infty}}{C_{\infty}}\right]
$$

have no zeros at $s=\infty$ iff

$$
\operatorname{rank}_{\mathbb{R}}\left[J_{\infty}, \bar{B}_{\infty}\right]=\mu \quad \text { and } \operatorname{rank}_{\mathbb{R}}\left[\begin{array}{c}
J_{\infty} \\
\bar{C}_{\infty}
\end{array}\right]=\mu .
$$

The next Proposition says that if the Smith-McMillan form $S_{A(s)}^{\infty}$ at $s=\infty$ of $A(s)$ is given by (2) and $C_{\infty}, A_{\infty}, B_{\infty}, D_{\infty}=0$ is a GSS realization of $A(s)$ which is obtained as in Remark 1 i.e., as a minimal state space realization of the strictly proper rational matrix $\bar{A}(s):=\frac{1}{s} A\left(\frac{1}{s}\right) \in \mathbb{R}_{p r}(s)^{p \times m}$, then this realization is an irreducible at $s=\infty$ GSS realization of $A(s)$ and the number $\eta$ of the Jordan blocks $J_{\infty i}$ in the Jordan form $J_{\infty}$ in (8) satisfies

$$
\eta=k,
$$

while the indices $\kappa_{i}$ of the sizes $\kappa_{i}+1, i=1,2, \ldots, \eta$ of the Jordan blocks $J_{\infty i}$ of $J_{\infty}$ in (9) are given by

$$
\kappa_{i}=q_{i}
$$

where $q_{i}>0, i=1,2, \ldots, k$ are the non-zero orders of the poles at $s=\infty$ appearing in the Smith-McMillan form $S_{A(s)}^{\infty}$ at $s=\infty$ of $A(s)$. This proposition also gives us necessary tools for the investigation of what constitutes a minimal GSS realization of a polynomial matrix $A(s)$.

Proposition 3. Let $A(s) \in \mathbb{R}[s]^{p \times m}$ with

$$
\operatorname{rank}_{\mathbb{R}(s)} A(s)=r
$$

and the Smith-McMillan form at $s=\infty$

$$
\begin{aligned}
S_{A(s)}^{\infty}= & \operatorname{diag}(\overbrace{s^{q_{1}}, \ldots, s^{q_{k}}, I_{v-k}}^{v}, \\
& \left.\frac{1}{s^{\widehat{q}_{v+1}}}, \ldots, \frac{1}{s^{\widehat{q}_{r}}}, 0_{p-r, m-r}\right),
\end{aligned}
$$

where $0 \leq k \leq v \leq r$, and $q_{1} \geq q_{2} \geq \cdots \geq q_{k}>0=$ $q_{k+1}=\cdots=q_{v}, \widehat{q}_{r} \geq \widehat{q}_{r-1} \geq \cdots \geq \widehat{q}_{v+1}>0$, are respectively the orders of the poles and zeros at $s=\infty$ of $A(s)$. Let also $C_{\infty} \in \mathbb{R}^{p \times \mu}, A_{\infty} \in \mathbb{R}^{\mu \times \mu}, B_{\infty} \in$
$\mathbb{R}^{\mu \times m}$ be a minimal state space realization of $\bar{A}(s) \in$ $\mathbb{R}_{p r}(s)^{p \times m}$, i.e.,

$$
\bar{A}(s):=\frac{1}{s} A\left(\frac{1}{s}\right)=C_{\infty}\left(s I_{\mu}-A_{\infty}\right)^{-1} B_{\infty},
$$

and let $J_{\infty}:=Q A_{\infty} Q^{-1},|Q| \neq 0$ be the Jordan normal form of $A_{\infty}$. Then

(i) the McMillan degree $\delta_{M}(\bar{A}(s))$ of the strictly proper rational matrix $\bar{A}(s)$ is given by

$$
\delta_{M}(\bar{A}(s))=\sum_{i=1}^{k} q_{i}+v ;
$$

(ii) we have

$$
\begin{aligned}
& J_{\infty} \\
& \quad=\text { block diag }\left[J_{\infty 1}, \ldots, J_{\infty k}, 0_{v-k, v-k}\right] \in \mathbb{R}^{\mu \times \mu},
\end{aligned}
$$

where

$$
J_{\infty i}=\left[\begin{array}{cccc}
0 & 1 & \ldots & 0 \\
\vdots & \vdots & \ddots & \vdots \\
0 & 0 & \ldots & 1 \\
0 & 0 & \ldots & 0
\end{array}\right] \in \mathbb{R}^{\left(q_{i}+1\right) \times\left(q_{i}+1\right),}
$$

$i=1,2, \ldots, k$;

(iii) $\left(C_{\infty}, A_{\infty}, B_{\infty}\right)$ or, equivalently, $\left(\bar{C}_{\infty}, J_{\infty}, \bar{B}_{\infty}\right)$ constitute an irreducible at $s=\infty$ GSS realization of $A(s)$.

Proof. Consider the strictly proper rational matrix

$$
\begin{aligned}
\bar{A}(s) & :=\frac{1}{s} A\left(\frac{1}{s}\right) \\
& =A_{0} \frac{1}{s}+A_{1} \frac{1}{s^{2}}+\cdots+A_{q_{1}} \frac{1}{s^{q_{1}+1}} \in \mathbb{R}_{p r}(s)^{p \times m} .
\end{aligned}
$$

Since $A(s)$ is a polynomial, all poles of $A(s)$ are located at $s=\infty$ and thus all poles of $\bar{A}(s)$ are at $s=0$. Furthermore, if $S_{\bar{A}(s)}^{0} \in \mathbb{R}(s)^{p \times m}$ is the (local) McMillan form of $\bar{A}(s)$ at $s=0$, then

$$
\begin{aligned}
& S_{\bar{A}(s)}^{0}(s)=\frac{1}{s} S_{A(s)}^{\infty}\left(\frac{1}{s}\right) \\
& =\operatorname{diag} \overbrace{\overbrace{v}^{\frac{1}{s^{q_{1}+1}}, \ldots, \frac{1}{s^{q_{k}+1}}}, \frac{1}{s} I_{v-k}}^{k}, \\
& \left.s^{\widehat{q}_{v+1}-1}, \ldots, s^{\widehat{q}_{r}-1}, 0_{p-r, m-r}\right) .
\end{aligned}
$$


Thus, if

$$
S \frac{\mathbb{C}}{A}(s)(s)=\operatorname{diag}\left[\frac{\epsilon_{1}(s)}{\psi_{1}(s)}, \ldots, \frac{\epsilon_{r}(s)}{\psi_{r}(s)}, 0_{p-r, m-r}\right]
$$

is the McMillan form (in $\mathbb{C}$ ) of $\bar{A}(s)$, then from (36) $\epsilon_{i}(s)=1, i=1,2, \ldots, v, \epsilon_{i}(s)=s^{\widehat{q}_{i}-1} e(s), e(s) \in$ $\mathbb{R}[s], e(0) \neq 0, i=v+1, \ldots, r$ and $\psi_{i}(s)=s^{q_{i}+1}$, $i=1,2, \ldots, k, \psi_{i}(s)=s, i=k+1, \ldots, v, \psi_{i}(s)=1$, $i=v+1, \ldots, r$. Now (i) follows from the fact that $\mu=\delta_{M}(\bar{A}(s)):=\sum_{i=1}^{v} \operatorname{deg} \psi_{i}(s)=\sum_{i=1}^{k}\left(q_{i}+1\right)+$ $v-k=\sum_{i=1}^{k} q_{i}+v$. Furthermore, if $C_{\infty} \in \mathbb{R}^{p \times \mu}$, $A_{\infty} \in \mathbb{R}^{\mu \times \mu}, B_{\infty} \in \mathbb{R}^{\mu \times m}$ is a minimal state space realization of $\bar{A}(s)$, then the Smith form (in $\mathbb{C}$ ) of $\left[s I_{\mu}-A_{\infty}\right]$ is given by

$$
\begin{aligned}
S_{\left[s I_{\mu}-A_{\infty}\right]}^{\mathbb{C}} & =\operatorname{diag}\left[I_{\mu-v}, \psi_{v}(s), \ldots, \psi_{1}(s)\right] \\
& =\operatorname{diag}\left[I_{\mu-v}, s I_{v-k}, s^{q_{k}+1}, \ldots, s^{q_{1}+1}\right] \\
& =S_{\left[s I_{\mu}-J_{\infty}\right]}^{\mathbb{C}}
\end{aligned}
$$

and (ii) follows from the definition of the Jordan normal form $J_{\infty}$ of $A_{\infty}$ via the non-trivial invariant polynomials $\psi_{i}(w), i=1,2, \ldots, v$ of $s I_{\mu}-A_{\infty}$ in (37). (iii) The fact that $C_{\infty}, A_{\infty}, B_{\infty}$ constitutes a GSS realization of $A(s)$ follows from (31) which, by the substitution $1 / s \rightarrow s$, yields (6) with $D_{\infty}=0$.

Now since $C_{\infty}, A_{\infty}, B_{\infty}$ is a minimal state space realization of $\bar{A}(s)$, it follows that

$$
\begin{aligned}
& \operatorname{rank}_{\mathbb{C}} {\left[s I_{\mu}-A_{\infty}, B_{\infty}\right] } \\
&=\operatorname{rank}_{\mathbb{C}}\left[\begin{array}{c}
s I_{\mu}-A_{\infty} \\
C_{\infty}
\end{array}\right]=\mu, \quad \forall s \in \operatorname{sp}\left(A_{\infty}\right),
\end{aligned}
$$

where $\mu=\delta_{M}(\bar{A}(s))$. Using the fact that $A_{\infty}$ has all its eigenvalues at $s=0$, it is easily seen that the above condition reduces to

$$
\operatorname{rank}_{\mathbb{R}}\left[A_{\infty}, B_{\infty}\right]=\operatorname{rank}_{\mathbb{R}}\left[\begin{array}{c}
A_{\infty} \\
C_{\infty}
\end{array}\right]=\mu .
$$

The fact that $C_{\infty}, A_{\infty}, B_{\infty}$ or, equivalently, $\bar{C}_{\infty}, J_{\infty}, \bar{B}_{\infty}$ are irreducible at $s=\infty$ GSS realizations of $A(s)$ follows from (38) and Proposition 2 .

Given a GSS realization $\Sigma_{g}=\left[C_{\infty} \in \mathbb{R}^{p \times \mu}, A_{\infty} \in\right.$ $\left.\mathbb{R}^{\mu \times \mu}, B_{\infty} \in \mathbb{R}^{\mu \times m}, D_{\infty} \in \mathbb{R}^{p \times m}\right], \mu \in Z^{+}$of a polynomial matrix $A(s) \in \mathbb{R}[s]^{p \times m}$, the above deliberations give rise to the next corollary which provides a relation between (i) the set of zeros at $s=\infty$ of the matrix pencil $I_{\mu}-s A_{\infty}$, (ii) the set of poles at $s=\infty$ of $A(s)$, and (iii) the set of decoupling zeros at $s=\infty$ of $\Sigma_{g}$.

\section{Corollary 4. There holds}

$$
\begin{aligned}
& \left\{\text { set of zeros at } s=\infty \text { of }\left[I_{\mu}-s A_{\infty}\right]\right\} \\
& \equiv\{\text { set of poles at } s=\infty \text { of } A(s)\} \\
& \quad \cup\left\{\text { set of decoupling zeros at } s=\infty \text { of } \Sigma_{g}\right\} .
\end{aligned}
$$

The above set relation gives rise to the equation

$$
\begin{aligned}
f_{g}:= & \left\{\# \text { of zeros at } s=\infty \text { of }\left[I_{\mu}-s A_{\infty}\right]\right\} \\
= & \{\# \text { of poles at } s=\infty \text { of } A(s)\} \\
& +\left\{\# \text { decoupling zeros at } s=\infty \text { of } \Sigma_{g}\right\} .
\end{aligned}
$$

Remark 2. Using (14), (40) can be written as

$$
f_{g}=\delta_{M}\left[I_{\mu}-s A_{\infty}\right]
$$

$$
=\delta_{M}(A(s))+\left\{\# \text { decoupling zeros at } s=\infty \text { of } \Sigma_{g}\right\} \text {. }
$$

Equation (40) gives rise to the inequality

$$
\begin{aligned}
f_{g} & :=\left\{\# \text { of zeros at } s=\infty \text { of }\left[I_{\mu}-s A_{\infty}\right]\right\} \\
& \geq\{\# \text { of poles at } s=\infty \text { of } A(s)\} \\
& =: \delta_{M}(A(s)) .
\end{aligned}
$$

If a GSS realization $\widehat{\Sigma}_{g}=\left[C_{\infty}, A_{\infty}, B_{\infty}, D_{\infty}\right]$ of a polynomial matrix $A(s)$ is irreducible at $s=\infty$, i.e., if $\widehat{\Sigma}_{g}$ has no i.d. and no o.d. zeros at $s=\infty$, then from Definition 3 we get

$$
\text { [\#decoupling zeros at } \left.s=\infty \text { of } \widehat{\Sigma}_{g}\right]=0,
$$

and from (42) the generalized order of $\widehat{\Sigma}_{g}$ takes its least the value of

$$
\begin{aligned}
\widehat{f_{g}} & =\delta_{M}\left[I_{\mu}-s A_{\infty}\right]=\operatorname{rank}_{\mathbb{R}} A_{\infty} \\
& =\operatorname{rank}_{\mathbb{R}} J_{\infty}=\sum_{i=1}^{k} q_{i}=: \delta_{M}(A(s)),
\end{aligned}
$$

which, by definition, is the McMillan degree $\delta_{M}(A(s))$ of $A(s)$. In such a case the irreducible at $s=\infty$ GSS realization $\widehat{\Sigma}_{g}=\left[C_{\infty}, A_{\infty},, B_{\infty}, D_{\infty}\right]$ of $A(s)$ has the least generalized order $\widehat{f}_{g}$ among the generalized orders of all GSS realizations which give rise to $A(s)$. As indicated by (43), the least generalized order $\widehat{f}_{g}$ of $\widehat{\Sigma}_{g}$ can then be determined directly from the McMillan degree of the polynomial matrix $A(s)$.

Definition 4, together with the above discussion and (42), gives rise to the following result.

Theorem 1. A GSS realization

$$
\widehat{\Sigma}_{g}=\left[C_{\infty}, A_{\infty}, B_{\infty}, D_{\infty}\right]
$$

of a polynomial matrix $A(s)$ with the Smith-McMillan form at $s=\infty$ as in (30) is irreducible at $s=\infty$ iff

$$
\begin{aligned}
\widehat{f_{g}} & :=\left\{\# \text { of zeros at } s=\infty \text { of }\left[I_{\mu}-s A_{\infty}\right]\right\} \\
& =\delta_{M}\left[I_{\mu}-s A_{\infty}\right]=\operatorname{rank}_{\mathbb{R}} A_{\infty} \\
& =\sum_{i=1}^{k} q_{1}=\delta_{M}(A(s)) .
\end{aligned}
$$


The next theorem is an analogue of Theorem 2.56 in (Vardulakis, 1991) for the case of irreducible at $s=\infty$ GSS realizations of a polynomial matrix $A(s)$.

Theorem 2. Let $A(s) \in \mathbb{R}[s]^{p \times m}$ with $\operatorname{rank}_{\mathbb{R}(s)} A(s)=$ $r$ and the Smith-McMillan form at $s=\infty$ as in (30).

Let $\widehat{\Sigma}_{g}=\left[C_{\infty} \in \mathbb{R}^{p \times \mu}, A_{\infty} \in \mathbb{R}^{\mu \times \mu}, B_{\infty} \in\right.$ $\left.\mathbb{R}^{\mu \times m}, D_{\infty} \in \mathbb{R}^{p \times m}\right], \mu \in \mathbb{Z}^{+}$, be an irreducible at $s=\infty$ GSS realization of $A(s)$. Then the zero structure at $s=\infty$ of $I_{\mu}-s A_{\infty}$ is isomorphic to the pole structure at $s=\infty$ of $A(s)$, i.e., if $S_{A(s)}^{\infty}$ is given by (30), then the Smith-McMillan form at $s=\infty$ of $I_{\mu}-s A_{\infty}$ is given by

$$
S_{\left[I_{\mu}-s A_{\infty}\right]}^{\infty}=\operatorname{diag}\left[s I_{\widehat{f}_{g}}, I_{v-k}, \frac{1}{s^{q_{k}}}, \ldots, \frac{1}{s^{q_{1}}}\right],
$$

where $\widehat{f}_{g}=\operatorname{rank}_{\mathbb{R}} A_{\infty}=\operatorname{rank}_{\mathbb{R}} J_{\infty}=\sum_{i=1}^{k} q_{i}=\mu-v$. Proof. Let $\Sigma_{g}=\left[C_{\infty}, A_{\infty}, B_{\infty}\right]$ be an irreducible at $s=$ $\infty$ GSS realization of $A(s)$ obtained as in Proposition 3 and let $J_{\infty}=Q A_{\infty} Q^{-1}$ be the Jordan normal form of $A_{\infty}$. Then the pole-zero structure at $s=\infty$ of $I_{\mu}-s A_{\infty}$ coincides with that of $I_{\mu}-s J_{\infty}$. From Proposition 3 , the form of $J_{\infty}$ in (33) and (34), 37) and since all finite zeros of $w I_{\mu}-J_{\infty}$ are at $w=0$, we have

$$
\begin{aligned}
S_{\left[w I_{\mu}-J_{\infty}\right]}^{\mathbb{C}} & =\operatorname{diag}\left[I_{\mu-v}, w I_{v-k}, w^{q_{k}+1}, \ldots, w^{q_{1}+1}\right] \\
& \equiv S_{\left[w I_{\mu}-J_{\infty}\right]}^{0}
\end{aligned}
$$

and therefore from Remark after Exercise 4.44 in Chapter 4 in (Vardulakis, 1991) we have

$$
\begin{aligned}
S_{\left[I_{\mu}-s A_{\infty}\right]}^{\infty} & \equiv S_{\left[I_{\mu}-s J_{\infty}\right]}^{\infty}=s S_{\left[w I_{\mu}-J_{\infty}\right]}^{0}\left(\frac{1}{s}\right) \\
& =\operatorname{diag}\left[s I_{\mu-\nu}, I_{v-k}, \frac{1}{s^{q_{k}}}, \cdots, \frac{1}{s^{q_{1}}}\right] .
\end{aligned}
$$

\section{Minimal GSS realizations of a polynomial matrix}

Now we discuss the concept of a minimal GSS realization of a polynomial matrix. Although the concepts of irreducibility $($ in $\mathbb{C}$ ) and minimality of a state space realization of a proper rational matrix coincide, i.e., irreducibility $($ in $\mathbb{C})$ of a state space realization of a proper rational matrix implies and is implied by minimality of the dimension of the state space realization, this is not in general true for the analogous concepts of irreducibility at $s=\infty$ and minimality of a GSS realization of a polynomial matrix. In the following, we first define what constitutes a minimal GSS realization of a polynomial matrix and then illustrate the above points by showing that minimality of a GSS realization of a polynomial matrix implies its irreducibility at $s=\infty$ (Theorem 3 ) but that in general the reverse is not true. Through these results we give necessary tools for obtaining (i) a minimal GSS realization of a polynomial matrix $A(s) \in \mathbb{R}[s]^{p \times m}$ and (ii) the least value of the dimension $\bar{\mu}$ of the generalized state vector $x_{\infty}(t) \in \mathbb{R}^{\bar{\mu}}$ appearing in a minimal GSS realization of $A(s)$.

Definition 5. A GSS realization $\widetilde{\Sigma}_{g}=\left[\widetilde{C}_{\infty} \in \mathbb{R}^{p \times \widetilde{\mu}}\right.$, $\widetilde{J}_{\infty} \in \mathbb{R}^{\tilde{\mu} \times \widetilde{\mu}}, \widetilde{B}_{\infty} \in \mathbb{R}^{\tilde{\mu} \times m}, \widetilde{D}_{\infty} \in \mathbb{R}^{p \times m}$ of a polynomial matrix $A(s) \in \mathbb{R}[s]^{p \times m}$ is called minimal if it has the least number of generalized states or, equivalently, if its dimension $\widetilde{\mu}$ is minimal, i.e., $\widetilde{\mu} \leq \mu$ for each dimension $\mu$ of all other GSS realizations

$$
\begin{aligned}
\Sigma_{g}= & {\left[C_{\infty} \in \mathbb{R}^{p \times \mu}, A_{\infty} \in \mathbb{R}^{\mu \times \mu}, B_{\infty} \in \mathbb{R}^{\mu \times m},\right.} \\
& \left.D_{\infty} \in \mathbb{R}^{p \times m}\right]
\end{aligned}
$$

of $A(s)$. The dimension $\widetilde{\mu}$ of a minimal GSS realization of a polynomial matrix $A(s)$ is called the least dimension of $A(s)$.

The next theorem gives a necessary and sufficient condition for a GSS realization of a polynomial matrix to be minimal.

Theorem 3. (Karampetakis, 1993) Let $A(s) \in \mathbb{R}[s]^{p \times m}$ with $\operatorname{rank}_{\mathbb{R}(s)} A(s)=r$ and the Smith-McMillan form at $s=\infty$ as in (2). Let also

$$
\begin{aligned}
\widetilde{\Sigma}_{g}= & {\left[\widetilde{C}_{\infty} \in \mathbb{R}^{p \times \widetilde{\mu}}, \widetilde{J}_{\infty} \in \mathbb{R}^{\tilde{\mu} \times \widetilde{\mu}}, \widetilde{B}_{\infty} \in \mathbb{R}^{\tilde{\mu} \times m},\right.} \\
& \left.\widetilde{D}_{\infty} \in \mathbb{R}^{p \times m}\right]
\end{aligned}
$$

be a GSS realization of $A(s)$ with $\widetilde{J}_{\infty}$ in the Jordan normal form and let $\bar{A}(s):=\frac{1}{s} A\left(\frac{1}{s}\right) \in \mathbb{R}_{p r}(s)^{p \times m}$. Then $\widetilde{\Sigma}_{g}=\left[\widetilde{C}_{\infty}, \widetilde{J}_{\infty}, \widetilde{B}_{\infty}, \widetilde{D}_{\infty}\right]$ is a minimal GSS realization of $A(s)$ iff

$$
\begin{aligned}
\widetilde{\mu}=\delta_{M}[\bar{A}(s)]- & (v-k) \\
& =\sum_{i=1}^{k}\left(q_{i}+1\right)=k+\sum_{i=1}^{k} q_{i} .
\end{aligned}
$$

Proof. We present the proof of (Karampetakis, 1993), which is constructive. As in Proposition 3, let $\bar{C}_{\infty} \in$ $\mathbb{R}^{p \times \mu}, J_{\infty} \in \mathbb{R}^{\mu \times \mu}, \bar{B}_{\infty} \in \mathbb{R}^{\mu \times m}$ be a minimal state space realization of the strictly proper rational matrix $\bar{A}(s):=\frac{1}{s} A\left(\frac{1}{s}\right) \in \mathbb{R}_{p r}(s)^{p \times m}$ with $J_{\infty}$ in the Jordan normal form as in (33), (34), and partition the matrices 


$$
\begin{aligned}
& \bar{C}_{\infty}, J_{\infty}, \bar{B}_{\infty} \text { as } \\
& J_{\infty}=\operatorname{block} \operatorname{diag}\left[\widetilde{J}_{\infty}, 0_{v-k, v-k}\right], \\
& \widetilde{J}_{\infty}=\text { block } \operatorname{diag}\left[J_{\infty 1}, J_{\infty 2}, \ldots, J_{\infty k}\right] \in \mathbb{R}^{\widetilde{\mu} \times \widetilde{\mu}}, \\
& J_{\infty i} \in \mathbb{R}^{\left(q_{i}+1\right) \times\left(q_{i}+1\right)}, i=1,2, \ldots, k, \\
& \bar{B}_{\infty}=:\left[\begin{array}{c}
\widetilde{B}_{\infty} \\
\widehat{B}_{\infty}
\end{array}\right], \quad \widetilde{B}_{\infty} \in \mathbb{R}^{\widetilde{\mu} \times m}, \\
& \widehat{B}_{\infty} \in \mathbb{R}^{(v-k) \times m}, \quad \bar{C}_{\infty}=:\left[\begin{array}{ll}
\widetilde{C}_{\infty} & \widehat{C}_{\infty}
\end{array}\right], \\
& \widetilde{C}_{\infty} \in \mathbb{R}^{p \times \widetilde{\mu}}, \quad \widehat{C}_{\infty} \in \mathbb{R}^{p \times(v-k)},
\end{aligned}
$$

where

$$
\widetilde{\mu}=\sum_{i=1}^{k}\left(q_{i}+1\right)=\mu-(v-k)
$$

Using the above partitioning and Proposition 3 (iii), we get

$$
\begin{gathered}
{\left[\begin{array}{cc}
\widetilde{J}_{\infty} & 0_{\widetilde{\mu}, v-k} \\
0_{v-k, \widetilde{\mu}} & 0_{v-k, v-k}
\end{array}\right]\left[\begin{array}{l}
\dot{x}_{1 \infty}(t) \\
\dot{x}_{2 \infty}(t)
\end{array}\right]} \\
=\left[\begin{array}{c}
x_{1 \infty}(t) \\
x_{2 \infty}(t)
\end{array}\right]-\left[\begin{array}{c}
\widetilde{B}_{\infty} \\
\widehat{B}_{\infty}
\end{array}\right] u(t), \\
y(t)=\left[\begin{array}{ll}
\widetilde{C}_{\infty} & \widehat{C}_{\infty}
\end{array}\right]\left[\begin{array}{l}
x_{1 \infty}(t) \\
x_{2 \infty}(t)
\end{array}\right]
\end{gathered}
$$

is an irreducible at $s=\infty$ GSS realization of $A(s)$. The vector $x_{2 \infty}(t)$ in (48) represents $v-k$ "non-dynamic" variables associated with the $(v-k) \times(v-k)$ zero block in $J_{\infty}$ and is related to the input $u(t)$ via $x_{2 \infty}(t)=$ $\widehat{B}_{\infty} u(t)$. Thus (48) and 49) can be written as

$$
\begin{aligned}
\widetilde{J}_{\infty} \dot{x}_{1 \infty}(t) & =x_{1 \infty}(t)-\widetilde{B}_{\infty} u(t) \\
y(t) & =\widetilde{C}_{\infty} x_{1 \infty}(t)+\widehat{C}_{\infty} x_{2 \infty}(t) \\
& =\widetilde{C}_{\infty} x_{1 \infty}(t)+\widetilde{D}_{\infty} u(t)
\end{aligned}
$$

where $\widetilde{D}_{\infty}:=\widehat{C}_{\infty} \widehat{B}_{\infty} \in \mathbb{R}^{p \times m}$. Recall that from Theo$\operatorname{rem} 1$ the condition $\operatorname{rank}_{\mathbb{R}} \widetilde{J}_{\infty}=\sum_{i=1}^{k} q_{1}=\delta_{M}(A(s))$ implies that the GSS realization $\widetilde{C}_{\infty} \in \mathbb{R}^{p \times \widetilde{\mu}}, \widetilde{J}_{\infty} \in$ $\mathbb{R}^{\tilde{\mu} \times \widetilde{\mu}}, \widetilde{B}_{\infty} \in \mathbb{R}^{\tilde{\mu} \times m}, \widetilde{D}_{\infty} \in \mathbb{R}^{p \times m}$ of $A(s)$ in (50), (51) is also an irreducible at $s=\infty$ GSS realization of $A(s)$. We will now show that it is also minimal.

Suppose now that there exists another irreducible at an $s=\infty$ GSS realization $\underline{C}_{\infty} \in \mathbb{R}^{p \times \bar{\mu}}, \underline{J}_{\infty} \in$ $\mathbb{R}^{\bar{\mu} \times \bar{\mu}}, \underline{B}_{\infty} \in \mathbb{R}^{\bar{\mu} \times m}, \underline{D}_{\infty} \in \mathbb{R}^{p \times m}$ of $A(s)$ with no nondynamic variables with

$$
S_{\left[I_{\bar{\mu}}-s \underline{J}_{\infty}\right]}^{\infty}=\operatorname{diag}\left[s I_{g}, I_{\bar{v}-\bar{k}}, \frac{1}{s^{\bar{q}_{k}}}, \frac{1}{s^{\bar{q}_{k-1}}}, \ldots, \frac{1}{s^{\bar{q}_{1}}}\right],
$$

where $\bar{q}_{i}>0, i=1,2, \ldots, \bar{k}$ are the orders of the zeros at $s=\infty$ of $\left[I_{\bar{\mu}}-s \underline{J}_{\infty}\right]$ and such that $\bar{\mu}<\tilde{\mu}$, or equivalently, such that

$$
\bar{\mu}=\sum_{i=1}^{\bar{k}}\left(\bar{q}_{i}+1\right)<\sum_{i=1}^{k}\left(q_{i}+1\right)=\widetilde{\mu} .
$$

Combining (42) and (52), we obtain

$$
\sum_{i=1}^{\bar{k}}\left(\bar{q}_{i}+1\right)=\sum_{i=1}^{\bar{k}} \bar{q}_{i}+\bar{k}<\sum_{i=1}^{k}\left(q_{i}+1\right) \leq \sum_{i=1}^{\bar{k}} \bar{q}_{i}+k
$$

which implies

$$
\bar{k}<k \text {. }
$$

But since the GSS realizations $\left[\widetilde{C}_{\infty} \in \mathbb{R}^{p \times \widetilde{\mu}}, \widetilde{J}_{\infty} \in\right.$ $\left.\mathbb{R}^{\tilde{\mu} \times \tilde{\mu}}, \widetilde{B}_{\infty} \in \mathbb{R}^{\tilde{\mu} \times m}, \widetilde{D}_{\infty} \in \mathbb{R}^{p \times m}\right]$ and $\left[\underline{C}_{\infty} \in\right.$ $\left.\mathbb{R}^{p \times \bar{\mu}}, \underline{J}_{\infty} \in \mathbb{R}^{\bar{\mu} \times \bar{\mu}}, \underline{B}_{\infty} \in \mathbb{R}^{\bar{\mu} \times m}, \underline{D}_{\infty} \in \mathbb{R}^{p \times m}\right]$ of $A(s)$ are both irreducible at $s=\infty$, according to Theorem 2 the pole structure at $s=\infty$ of $A(s)$ is isomorphic to the zero structure at $s=\infty$ of $\left[I_{\widetilde{\mu}}-s \widetilde{J}_{\infty}\right]$ and $\left[I_{\bar{\mu}}-s \underline{J}_{\infty}\right]$ (42), and thus

$$
\begin{aligned}
\bar{k} & =\emptyset \text { of zeros at } s=\infty \text { of }\left[I_{\bar{\mu}}-s \underline{J}_{\infty}\right] \\
& =\emptyset \text { of poles at } s=\infty \text { of } A(s)=k,
\end{aligned}
$$

where the symbol ' $q$ ' denotes the total number of zeros at $s=\infty$ without counting orders. From (55) we can see that (54) is not true, which contradicts our assumption that there exists a GSS realization $\underline{C}_{\infty} \in \mathbb{R}^{p \times \bar{\mu}}, \underline{J}_{\infty} \in$ $\mathbb{R}^{\bar{\mu} \times \bar{\mu}}, \underline{B}_{\infty} \in \mathbb{R}^{\bar{\mu} \times m}, \underline{D}_{\infty} \in \mathbb{R}^{p \times m}$ of $A(s)$ satisfying $\bar{\mu}<\widetilde{\mu}$.

Remark 3. Two important conclusions that result from Theorem 3 are the following:

(i) A necessary condition for a GSS realization

$$
\begin{aligned}
& \widetilde{\Sigma}_{g}= {\left[\widetilde{C}_{\infty} \in \mathbb{R}^{p \times \widetilde{\mu}}, \widetilde{J}_{\infty} \in \mathbb{R}^{\tilde{\mu} \times \tilde{\mu}}, \widetilde{B}_{\infty} \in \mathbb{R}^{\tilde{\mu} \times m},\right.} \\
&\left.\widetilde{D}_{\infty} \in \mathbb{R}^{p \times m}\right]
\end{aligned}
$$

of a polynomial matrix $A(s)$ to be minimal is that the realization must be irreducible at $s=\infty$ and that the realization has no non-dynamic variables.

(ii) The least dimension that a GSS realization of $A(s)$ may have is $\widetilde{\mu}=\sum_{i=1}^{k}\left(q_{i}+1\right)$, where $q_{i}>0, i=$ $1,2, \ldots, k$ are the nonzero orders of the poles at $s=$ $\infty$ of $A(s)$.

Notice that if $v=k$, i.e., if in (48) there are not "nondynamic variables" (equivalently if there is no $I_{v-k}$ block in (30), then $\widetilde{\mu} \equiv \mu$ and a minimal state space realization $C_{\infty} \in \mathbb{R}^{p \times \mu}, J_{\infty} \in \mathbb{R}^{\mu \times \mu}, B_{\infty} \in \mathbb{R}^{\mu \times m}$ of $\bar{A}(s)$ $\in \mathbb{R}_{p r}(s)^{p \times m}$ coincides with a minimal GSS realization $\widetilde{C}_{\infty} \in \mathbb{R}^{p \times \widetilde{\mu}}, \widetilde{J}_{\infty} \in \mathbb{R}^{\tilde{\mu} \times \tilde{\mu}}, \widetilde{B}_{\infty} \in \mathbb{R}^{\tilde{\mu} \times m}, \widetilde{D}_{\infty} \in \mathbb{R}^{p \times m}$ of $A(s) \in \mathbb{R}[s]^{p \times m}$. 
Corollary 5. A GSS realization of a polynomial matrix $A(s)$ is a minimal GSS realization if it is an irreducible at $s=\infty$ GSS realization and has no non-dynamic variables.

For conventional state space realizations of a proper rational matrix $A(s)$, the least possible dimension of a state space realization is known as the least order $\nu(A(s))$ of $A(s)$ (Rosenbrock, 1970). The next remark gives an algorithm for the computation of the least dimension $\widetilde{\mu}$ of a minimal GSS realization of a polynomial matrix.

Remark 4. (Karampetakis, 1993) The least dimension $\widetilde{\mu}=\sum_{i=1}^{k}\left(q_{i}+1\right)$ of a minimal GSS realization of a polynomial matrix $A(s) \in \mathbb{R}^{p \times m}[s]$ can be calculated without computing the orders of the poles at $s=\infty$ in the Smith-McMillan form at $s=\infty$ of $A(s)$. Let

$$
\mathcal{X}_{p}=\left[\begin{array}{cccc}
A_{q_{1}} & 0 & \cdots & 0 \\
\vdots & A_{q_{1}} & \ddots & 0 \\
A_{p+1} & \ddots & \ddots & \vdots \\
A_{p} & A_{p+1} & \cdots & A_{q_{1}}
\end{array}\right]
$$

$p=0,1, \ldots, q_{1}$. From Exercise 3.89 in (Vardulakis, 1991), we have

$$
\begin{aligned}
\delta_{M}(A(s)) & =\operatorname{rank} \mathcal{X}_{1} \\
& =\delta_{M}\left(A_{1} s+A_{2} s^{2}+\cdots+A_{q_{1}} s^{q_{1}}\right) .
\end{aligned}
$$

Applying the same result to $\mathcal{X}_{2}$, we get

$$
\begin{aligned}
\operatorname{rank}_{\mathbb{R}} \mathcal{X}_{2} & =\delta_{M}\left(A_{2} s+A_{3} s^{2}+\cdots+A_{q_{1}} s^{q_{1}-1}\right) \\
& =\delta_{M}\left(A_{1}+A_{2} s+\cdots+A_{q_{1}} s^{q_{1}-1}\right) \\
& =\delta_{M}\left(\frac{A_{1} s+A_{2} s^{2}+\cdots+A_{q_{1}} s^{q_{1}}}{s}\right) \\
& =\delta_{M}\left(\frac{1}{s} A(s)\right) .
\end{aligned}
$$

Since $A(s)$ has only poles at $s=\infty$, it can be easily seen that

$$
\delta_{M}\left(\frac{1}{s} A(s)\right)=\sum_{i=1}^{k}\left(q_{i}-1\right)=\delta_{M}(A(s))-k .
$$

From (57) the number $k$ of strictly positive orders $q_{i}>0$ of poles at $s=\infty$ of $A(s)$ is given by

$$
k=\delta_{M}(A(s))-\delta_{M}\left(\frac{1}{s} A(s)\right),
$$

which, due to (56), gives

$$
k=\operatorname{rank} \mathcal{X}_{1}-\operatorname{rank}_{\mathbb{R}} \mathcal{X}_{2}
$$

and, therefore,

$$
\widetilde{\mu}=2 \operatorname{rank} \mathcal{X}_{1}-\operatorname{rank}_{\mathbb{R}} \mathcal{X}_{2} .
$$

If $A(s)$ is a $p \times m$ column (row) proper polynomial matrix with $p \geq m(p \leq m)$, then an irreducible at $s=\infty$ GSS realization of $A(s)$ as well as the SmithMcMillan form at $s=\infty$ of $A(s)$ can be obtained by inspection. This is stated in the following result.

Proposition 4. Let $A(s) \in \mathbb{R}[s]^{p \times m}, p \geq m$, be column proper and let $v_{i}=\operatorname{deg} a_{j}(s)$, where $a_{j}(s)=a_{j 0}+$ $a_{j 1} s+\cdots+a_{j v_{j}} s^{v_{j}} \in \mathbb{R}[s]^{p \times 1}, j=1,2, \ldots, m$ are the $m$ columns of $A(s)$. Then an irreducible at $s=\infty$ GSS realization $C_{\infty}, J_{\infty}, B_{\infty}$ of $A(s)$ can be obtained by inspection and is given by

$$
\begin{gathered}
C_{\infty}=\left[a_{1 v_{1}}, \cdots, a_{10}|\cdots| a_{m v_{m}}, \cdots, a_{m 0}\right] \in \mathbb{R}^{p \times \mu}, \\
J_{\infty}=\text { block diag }\left[J_{\infty 1}, J_{\infty 2}, \cdots, J_{\infty m}\right] \in \mathbb{R}^{\mu \times \mu}, \\
J_{\infty j}=\left[\begin{array}{cccc}
0 & 1 & \ldots & 0 \\
\vdots & \vdots & \ddots & \vdots \\
0 & 0 & \ldots & 1 \\
0 & 0 & \ldots & 0
\end{array}\right] \in \mathbb{R}^{\left(v_{i}+1\right) \times\left(v_{i}+1\right)} \\
B_{\infty}=\text { block diag }\left[b_{1}, b_{2}, \ldots, b_{m}\right] \in \mathbb{R}^{\mu \times m} \\
b_{j}=[0,0, \ldots, 0,1]^{\top} \in \mathbb{R}^{\left(v_{j}+1\right) \times 1}
\end{gathered}
$$

where $j=1,2, \ldots, m$ and $\mu=\sum_{j=1}^{m}\left(v_{j}+1\right)$.

Proof. Write $A(s)=[A(s)]_{c}^{h} \operatorname{diag}\left(s^{v_{1}}, \ldots s^{v_{m}}\right)+$ $A_{1} \bar{S}(s)$, where $[A(s)]_{c}^{h}$ denotes the highest column degree coefficient matrix of the matrix inside the brackets and

$$
\bar{S}(s)=\text { block } \operatorname{diag}\left\{\left[\begin{array}{c}
s^{v_{1}-1} \\
\vdots \\
s \\
1
\end{array}\right], \cdots,\left[\begin{array}{c}
s^{v_{m}-1} \\
\vdots \\
s \\
1
\end{array}\right]\right\},
$$

$A_{1} \in \mathbb{R}^{p \times \mu}$. Then

$$
\begin{aligned}
& \frac{1}{w} A\left(\frac{1}{w}\right) \\
= & {[A(s)]_{c}^{h} \operatorname{diag}\left(\frac{1}{w^{v_{1}+1}}, \ldots, \frac{1}{w^{v_{m}+1}}\right)+\frac{1}{w} A_{1} \bar{S}\left(\frac{1}{w}\right) } \\
= & {[A(s)]_{c}^{h}+\frac{1}{w} A_{1} \bar{S}\left(\frac{1}{w}\right) \operatorname{diag}\left(w^{v_{1}+1}, \ldots, w^{v_{m}+1}\right) } \\
& \times\left[\operatorname{diag}\left(w^{v_{1}+1}, \ldots, w^{v_{m}+1}\right)\right]^{-1} \\
= & N(w) D(w)^{-1},
\end{aligned}
$$

where $N(w) \in \mathbb{R}[w]^{p \times m}$ can also be written as

$$
N(w)=C_{\infty} S(w)
$$

with

$$
S(w)=\text { block } \operatorname{diag}\left\{\left[\begin{array}{c}
1 \\
w \\
\vdots \\
w^{v_{1}}
\end{array}\right], \ldots,\left[\begin{array}{c}
1 \\
w \\
\vdots \\
w^{v_{m}}
\end{array}\right]\right\} .
$$


Now, since for $w=0$,

$$
\operatorname{rank}\left[\begin{array}{c}
D(0) \\
N(0)
\end{array}\right]=\operatorname{rank}\left[\begin{array}{c}
0_{m, m} \\
{[A(s)]_{c}^{h}}
\end{array}\right]=m
$$

and for $0 \neq w \in \mathbb{C}$,

$$
\operatorname{rank}_{\mathbb{C}}\left[\begin{array}{c}
D(w) \\
N(w)
\end{array}\right]=m
$$

we have that the expression in 66 is a right coprime MFD of $\frac{1}{w} A\left(\frac{1}{w}\right) \in \mathbb{R}_{p r}(s)^{p \times m}$ with $D(w)$ column proper. Thus, $C_{\infty}, J_{\infty}, B_{\infty}$ is a minimal state space realization of $\frac{1}{w} A\left(\frac{1}{w}\right)$.

Remark 5. A dual result can be obtained for every $p \times m(p \leq m)$ row proper polynomial matrix $A(s)$ by transposing $A(s)$ and applying Remark 3. Comparing the dimensions of the Jordan blocks $J_{\infty i}$ in (34) of Proposition 3 and those of the Jordan blocks in (62) of Proposition 4, we can easily conclude that if $A(s)$ is column (row) proper, then its column (row) degrees $v_{j}, j=1,2, \ldots, m$ $\left(r_{i}, i=1,2, \ldots, p\right)$ are equal to the orders of its poles at $s=\infty$, i.e., that $v_{j} \equiv q_{j}, j=1,2, \ldots, m\left(r_{i} \equiv q_{i}\right.$, $i=1,2, \ldots, p)$ and that $A(s)$ has no zeros at $s=\infty$.

\section{Conclusions}

In this paper we have discussed the concepts of irreducibility and minimality of "pure" generalized state space realizations of polynomial transfer function matrices. Our main focus was to point out the difference between these two concepts. Irreducibility of "pure" generalized state space systems was shown to be associated with the absence of decoupling zeros at infinity, and the cancellation mechanism of zeros at infinity was clearly explained. On the other hand, it turned out that a generalized state space realization had minimal state dimension if it was irreducible (at infinity) and had no non-dynamic variables. Furthermore, we proposed both a method for the determination of the minimal state dimension and the construction of a minimal "pure" generalized state space realization.

\section{References}

Bosgra, O. and Van Der Weiden, A. (1981). Realizations in generalized state-space form for polynomial system matrices and the definitions of poles, zeros and decoupling zeros at infinity, International Journal of Control 33(3): 393-411.

Christodoulou, M. and Mertzios, B. (1986). Canonical forms for singular systems, Proceedings of the of 25th IEEE Conference on Decision and Control (CDC), Athens, Greece, pp. 2142-2143.

Cobb, D. (1984). Controllability, observability, and duality in singular systems, IEEE Transactions on Automatic Control 29(12): 1076-1082.
Conte, G. and Perdon, A. (1982). Generalized state space realizations of non-proper rational transfer functions, Systems and Control Letters 1(4): 270-276.

Gantmacher, F. (1959). The Theory of Matrices, Chelsea Publishing Company, New York, NY.

Karampetakis, N. (1993). Notions of Equivalence for Linear Time Invariant Multivariable Systems, Ph.D. thesis, Department of Mathematics, Aristotle University of Thessaloniki.

Lewis, F. (1986). A survey of linear singular systems, Circuits, Systems, and Signal Processing 5(1): 3-36.

Lewis, F., Beauchamp, G. and Syrmos, V. (1989). Some useful aspects of the infinite structure in singular systems, Proceedings of the International Symposium MTNS-89, Amsterdam, The Netherlands, pp. 263-270.

Misra, P. and Patel, R. (1989). Computation of minimal-order realizations of generalized state-space systems, Circuits, Systems, and Signal Processing 8(1): 49-70.

Rosenbrock, H. (1970). State Space and Multivariable Theory, Nelson, London.

Rosenbrock, H. (1974). Structural properties of linear dynamical systems, International Journal of Control 20(2): 191-202.

Vafiadis, D. and Karcanias, N. (1995). Generalized state-space realizations from matrix fraction descriptions, IEEE Transactions on Automatic Control 40(6): 1134-1137.

Vardulakis, A. (1991). Linear Multivariable Control: Algebraic Analysis and Synthesis Methods, Wiley, New York, NY.

Vardulakis, A. and Karcanias, N. (1983). Relations between strict equivalence invariants and structure at infinity of matrix pencils, IEEE Transactions on Automatic Control 28(4): 514-516.

Vardulakis, A., Limebeer, D. and Karcanias, N. (1982). Structure and Smith-MacMillan form of a rational matrix at infinity, International Journal of Control 35(4): 701-725.

Varga, A. (1989). Computation of irreducible generalized statespace realizations, Kybernetika 26(2): 89-106.

Verghese, G. (1978). Infinite Frequency Behavior in Dynamical Systems, Ph.D. thesis, Department of Electrical Engineering, Stanford University.

Verghese, G., Levy, B. and Kailath, T. (1981). A generalized state-space for singular systems, IEEE Transactions on Automatic Control 26(4): 811-831. 


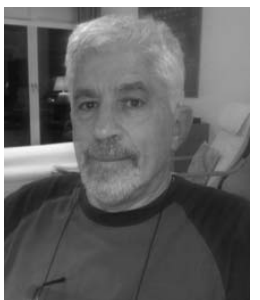

Antonis - Ioannis G. Vardulakis received the B.Sc. degree in physics from the University of Athens, Greece, and the M.Sc and $\mathrm{Ph} . \mathrm{D}$. degrees in automatic control from the Control Systems Centre of the University of Manchester Institute of Science and Technology (UMIST), Manchester, UK, in 1969, 1971 and 1974, respectively. From 1975 until 1978 he was employed as a research assistant at the Department of Computing and Control, Imperial College of Science and Technology, London, UK, and from 1978 until 1985 he worked for the Control and Management System Division of the Engineering Department, University of Cambridge, as a research scientist. In 1984 he was appointed a professor of systems and control theory at the Department of Mathematics of the Aristotle University of Thessaloniki, Greece. He has published more than 150 research and conference papers and in 1991 he published the monograph entitled Linear Multivariable Control: Algebraic Analysis and Synthesis Methods. His research interests include mathematical systems and control theory, multivariable control, numerical and symbolic methods for computer aided analysis and design of control systems. He is a senior member of the IEEE and a founding member of the European Union Control Association (EUCA)

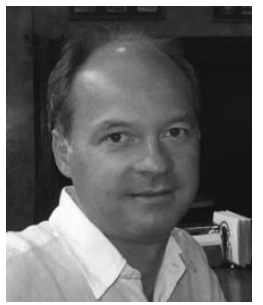

Nicholas P. Karampetakis received his B.Sc. degree in mathematics in 1989 and the Ph.D. degree in mathematics in 1993 at the Department of Mathematics of the Aristotle University of Thessaloniki, Greece. From 1994 till 1995, he was a research associate at the Department of Mathematics of Loughborough University of Technology, UK. From 1995 till 2000, he was a research assistant at the Department of Mathematics of the Aristotle University of Thessaloniki. Since 2000, he has been holding the position of an assistant professor of control theory at the Department of Mathematics of the Aristotle University of Thessaloniki. He is an author/co-author of more than 40 papers published in international journals and of more than 60 papers that have appeared at international conferences. He has authored one book, entitled Introduction to Fortran 90/95, and co-authored one book, entitled Mathematics and Programming in Mathematica (both in Greek). His research interests are in the area of mathematical systems theory and include the algebraic-polynomial approach to the analysis and synthesis of linear multivariable systems and multi-dimensional (nD) systems. He is a member of the IFAC Technical Committee on Linear Systems, and a Vice-Chair of the IEEE Action Group on Symbolic Methods for CACSD

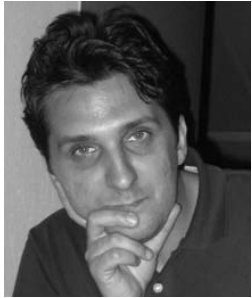

Efstathios N. Antoniou received his B.Sc. degree in mathematics in 1994 and his Ph.D. in mathematical systems theory in 2000, both from the Department of Mathematics of the Aristotle University of Thessaloniki, Greece. During the last decade he has been employed as a researcher in several research projects in the area of Computer Aided Control System Design (CACSD). In 2008, he was appointed an assistant professor of applied mathematics at the Technical Educational Institute of Thessaloniki. His research and teaching interests lie in the area of polynomial description of linear mult variable systems, systems stability, numerical linear algebra, and the implementation of numerical and symbolic software for control theory.

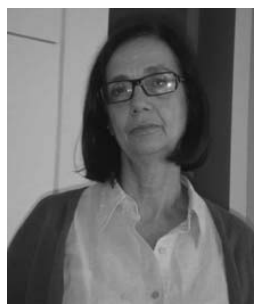

Evangelia Tictopoulou received the B.Sc. degree in mathematics from the University of Athens, Greece, in 1972, and the M.Sc. degree with specialization in automatic calculation from the University of Pisa, Italy, in 1975. From 1977 till 1989 she worked as an analystprogrammer in private enterprises. In 1989, she was appointed an assistant professor of computer science at the General Department of Applied Science of the Technical Educational Institute of Chalkis, Greece. Her main interest is in the area of genetic algorithms.

Received: 15 February 2008 